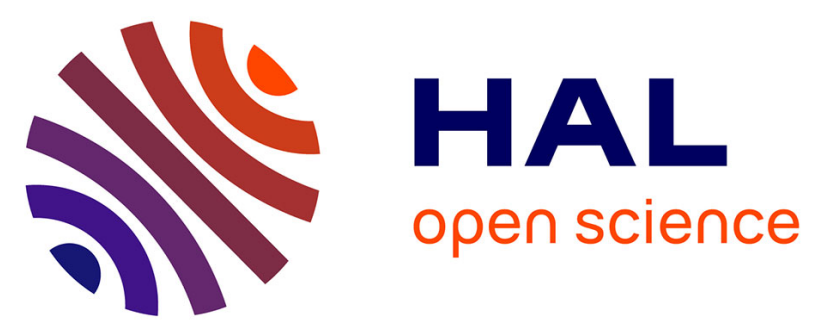

\title{
Organogenèse et croissance du maître-brin du blé tendre (Triticum aestivum) du semis à la floraison. II. - Contrôle des relations entre la croissance et la vascularisation de la tige et des feuilles. Essai de modélisation
}

\author{
Pierre Malvoisin
}

\section{To cite this version:}

Pierre Malvoisin. Organogenèse et croissance du maître-brin du blé tendre (Triticum aestivum) du semis à la floraison. II. - Contrôle des relations entre la croissance et la vascularisation de la tige et des feuilles. Essai de modélisation. Agronomie, 1984, 4 (7), pp.587-596. hal-00884676

\section{HAL Id: hal-00884676 https://hal.science/hal-00884676}

Submitted on 1 Jan 1984

HAL is a multi-disciplinary open access archive for the deposit and dissemination of scientific research documents, whether they are published or not. The documents may come from teaching and research institutions in France or abroad, or from public or private research centers.
L'archive ouverte pluridisciplinaire HAL, est destinée au dépôt et à la diffusion de documents scientifiques de niveau recherche, publiés ou non, émanant des établissements d'enseignement et de recherche français ou étrangers, des laboratoires publics ou privés. 


\section{Organogenèse et croissance du maître-brin du blé tendre (Triticum aestivum) du semis à la floraison. II. - Contrôle des relations entre la croissance et la vascularisation de la tige et des feuilles. Essai de modélisation.}

Pierre MALVOISIN ( $\left.{ }^{1}\right)$

I.N.R.A., Station d'Amélioration des Plantes, BV 1540, F 21034 Dijon

RÉSUMÉ

On étudie l'organogenèse et la croissance des parties aériennes du maître-brin du blé (Triticum aestivum), en mesurant la longueur de tous les organes depuis leur initiation jusqu'à la fin de leur expansion. On met en relation l'organisation de la croissance avec la mise en place du système vasculaire de la tige. Deux pôles d'activité sont mis en évidence : (1) le dôme terminal, qui initie les phytomères, relayé, lorsque le dernier phytomère sera créé (épillet terminal), par la population des bourgeons d'épillets de l'épi ; (2) un ensemble de quatre positions $\alpha, \beta, \gamma, \delta$, où se déroule exclusivement la croissance effective des organes : $\alpha$ correspond au nœud en provascularisation porteur du limbe enroulé non visible, $\beta$, au nœud en vascularisation porteur du limbe qui émerge, $\gamma$, au nœud pleinement vascularisé porteur de la gaine qui achève son expansion et qui entoure le limbe $\alpha$ et la gaine $\beta$ non visibles, $\delta$, au nœud qui s'épaissit ; sous la position $\delta$ il n'y a plus de croissance des organes. Entre ces deux pôles d'activité, se constitue une zone de phytomères en attente (zone $\mu$ ) dont la croissance est de type modéré.

L'activité de croissance localisée dans l'ensemble $\alpha, \beta, \gamma, \delta$ se déplace de manière acropète, remontant régulièrement, nœud après nœud, la plante. C'est l'émergence du limbe $\alpha$ et de la gaine $\beta$ hors du tube formé par la gaine $\gamma$ qui semble constituer l'événement responsable de la translation du système. Cette activité paraît contrôler l'activité organogène du dôme et des bourgeons d'épillet. On propose, en conclusion, une approche de modélisation du fonctionnement intégré de la plante dans cette première partie du cycle de morphogenèse.

Mots clés additionnels : Développement, corrélations, modélisation.

Organogenesis and growth of the main culm of Triticum aestivum from sowing to flowering. II. - An attempted model of the relation between growth and vascularization of culm and leaves.

Studies of the growth and development of the main culm of wheat were conducted by measuring the length of all organs from initiation to maturity in relation with the vascular development of the culm. Growth was localized in two active regions : (i) the shoot apical meristem, initiating the phytomers, then the population of spikelet bud meristems after the initiation of the last phytomer (terminal spikelet) ; (ii) a set of four positions $\alpha, \beta, \gamma, \delta$ where the only effective growth is occurring : $\alpha$ corresponds to the provascularized node, bearing the rolled-up lamina still not visible $; \beta$, to the vascularizing node, bearing the unfolding lamina $; \gamma$ to the fully vascularized node, bearing the sheath surrounding the hidden lamina $\alpha$ and sheath $\beta ; \delta$ corresponds to the just visible and thickening node; below $\delta$, no more growth occurs. Between these active regions, a piled structure of phytomers is set up, with only moderate growth rate ( $\mu$ region).

This process of growth, located in the $\alpha, \beta, \gamma, \delta$ region, moves upwards, node after node, as the plant develops and strongly influences the activity of the shoot apical and spikelet bud meristems. The data suggested that appearance of the lamina $\alpha$ and sheath $\beta$ from the sheath $\gamma$ determines the translation of the system. An integrated pattern of wheat growth and development from seeding to heading stages is discussed.

Additional key-words : Development, correlations, modelisation.

\section{INTRODUCTION}

L'étude de la morphogenèse d'un végétal ne se limite pas à la seule description des stades phénologi-

(i) Adresse actuelle : CIMMYT, Londres 40, Colonia Juarez, Delegation Cuauthtémoc, Appartado postal 6-641, 06600 Mexico DF, Mexique. ques ou à l'évolution quantitative de la croissance. Du dôme apical au système radiculaire, une plante est constituée d'un empilement d'organes; chaque organe, sous l'influence du milieu, suit une propre évolution en étroite coordination avec le reste de la plante (NOZERAN, 1978).

Le travail que nous présentons s'inscrit dans le cadre d'une approche de type cybernétique de la mor- 
phogenèse du blé, limitée aux seules parties aériennes du maître-brin. Comment s'effectue le développement intégré de la plante, quelle est la nature des liaisons entre les différentes parties du système?, telles sont les questions soulevées ici. Dans un précédent article (Malvoisin, 1984), on a tracé la trame du déroulement d'un parcours type; la régularité du rythme d'émergence des feuilles en fonction de la somme des températures moyennes journalières (base $0{ }^{\circ} \mathrm{C}$ ) est apparue comme une propriété générale. La croissance de la plante s'effectue étape par étape ; la durée séparant l'émergence de 2 feuilles successives, ou thermochrone, donne la dimension d'une étape élémentaire de croissance. L'émergence régulière des limbes est le signe qu'une activité ordonne la croissance des organes de manière stricte : il apparaît nécessaire de préciser le fonctionnement de cette activité de croissance. Pour cela, on a cherché à comprendre l'organisation de la croissance en longueur de tous les organes, depuis leur initiation jusqu'à leur pleine expansion, en relation avec la mise en place du système vasculaire.

\section{MATÉRIEL ET MÉTHODE}

On s'est intéressé d'abord à une situation particulière, ayant valeur de situation de référence. Puis on a élargi les investigations à un grand nombre d'autres situations pour vérifier la validité des observations. On ne rapporte dans ce travail que les résultats relatifs à la situation de référence et ayant valeur de généralisation.

\section{A. Matériel}

Les semences d'un blé alternatif (mutant $n^{\circ} 239$ du blé d'hiver "Capelle ») ont été mises à germer dans une étuve à germination $\left(20^{\circ} \mathrm{C}\right)$ pendant $36 \mathrm{~h}$, après désinfection à l'hypochlorite de calcium. On a repiqué les grains germés présentant une même longueur de coléoptile dans des pots de $15 \times 15 \times 15 \mathrm{~cm}$, remplis d'un mélange standard de tourbe et gravier, à raison de 4 grains par pot. On a ainsi semé 48 pots. L'expérience a été conduite en chambre climatisée, réglée à $20^{\circ} \mathrm{C}$ et $16 \mathrm{~h}$ d'éclairement pour une intensité de $70 \mathrm{wm}^{-2}$ environ, assurant un développement satisfaisant des plantes. Les pots ont été arrosés régulièrement avec une solution nutritive équilibrée standard.

\section{B. Méthode}

\section{Définitions}

Nous rappelons d'abord la signification que nous prêtons aux termes suivants :

\section{a) Stade foliaire}

C'est le nombre de feuilles pleinement sorties, la feuille en expansion étant affectée d'une décimale correspondant à son degré d'avancement à partir de son émergence.

\section{b) Thermochrone}

L'avancement du stade foliaire en fonction de la somme des températures moyennes journalières (base $0^{\circ} \mathrm{C}$ ) est très bien ajusté par une régression linéaire. On calcule le thermochrone, durée exprimée en nombre de degrés-jours, séparant l'émergence de 2 feuilles successives; le parcours de la plante s'effectue étape par étape, le thermochrone représente la durée d'une étape élémentaire de croissance ; le thermochrone est, en moyenne, constant tout au long du parcours et sa valeur est spécifique à chaque expérimentation (en général $100^{\circ} \mathrm{C}$ ). Si le thermochrone est constant, il n'en est pas de même du plastochrone, durée séparant l'initiation de 2 initiums successifs. Par souci de clarté, nous préférons utiliser la notion de rythme d'initiation par thermochrone pour caractériser l'activité organogène apicale.

\section{c) Phytomère}

L'activité du dôme apical est discrète. Périodiquement, l'apex produit un massif cellulaire, destiné à évoluer en organes qui auront le même âge, que nous convenons d'appeler phytomère. Un phytomère pourra évoluer en un ensemble limbe, gaine, nœud porteur de la feuille et entre-nœud situé sous le nœud (éventuellement), nous parlerons dans ce cas de phytomère végétatif, ou bien en un ensemble épillet, nœud de rachis et entre-nœud, nous aurons affaire alors à un phytomère floral ; l'ensemble des phytomères floraux forme l'épi.

Un phytomère correspond à une unité de position : on affecte un numéro à chaque phytomère selon son ordre d'apparition, le $1^{\text {er }}$ phytomère correspondant à la $1^{\text {re }}$ feuille. La plante est formée d'un empilement de phytomères que l'on peut regrouper en ensembles comme nous le verrons.

\section{d) Période juvénile, transitoire, florale}

L'observation de l'activité organogène apicale permet de scinder le parcours semis-épiaison en 3 périodes :

- une période juvénile où l'apex initie les phytomères végétatifs au rythme régulier de 2 phytomères par thermochrone;

- une période transitoire où corrélativement à l'apparition des $1^{\text {res }}$ ébauches d'entre-nœuds, le rythme d'initiation s'accélère ; ainsi se mettent en place les phytomères floraux qui donneront le futur épi. C'est aussi la période de tallage herbacé ;

- enfin une période florale où chaque bourgeon d'épillet évolue floralement, de manière régulière (un pas floral par thermochrone) jusqu'à l'épiaison. Cette période correspond à la montaison. L'échelle des stades phénologiques servant de repères a été antérieurement publiée (MALVOISIN, 1984, cf. annexe).

\section{Mesures effectuées}

Le stade foliaire de 8 plantes témoins, choisies au hasard, est suivi tout au long du parcours. Régulièrement on prélève 3 ou 4 plantes dont le stade foliaire est proche du stade foliaire moyen des plantes témoins. Les plantes choisies sont disséquées en découpant, selon le plan médian longitudinal, une tranche fine de moins d'1 $\mathrm{mm}$ d'épaisseur, de 
manière à observer la zone sous-apicale tout en laissant intact le dôme terminal ou le jeune épi, lorsque celui-ci se constitue ; la préparation, montée sur une lame, est observée immédiatement à la loupe binoculaire ou au microscope. Certaines préparations, fixées et colorées pendant quelques secondes au carmin acétique, ont été conservées dans du baume du Canada. Les observations ont porté sur :

a) la mesure de la longueur de tous les organes depuis leur initiation jusqu'à la fin de leur expansion ;

b) une estimation de l'état de vascularisation des nœuds.

Les mesures ayant débuté quelques jours après le repiquage, les phytomères d'ordre 1 et 2 ont été exclus de l'analyse.

Les résultats individuels (et non les moyennes) sont rapportés à l'échelle de temps biologique, c'est-à-dire en « stade foliaire ». Le passage du temps biologique au temps calendaire est donné par la régression du stade foliaire moyen des 8 plantes témoins en fonction de la somme des températures moyennes journalières (base $0{ }^{\circ} \mathrm{C}$ ) depuis le semis. Les courbes de croissance ainsi obtenues sont interprétées graphiquement selon une méthode explicitée en annexe.

\section{RÉSULTATS}

\section{A. Analyse de la croissance en longueur des limbes}

Pour mettre en évidence la coordination de la croissance intra- et inter-phytomères, on a étudié au préalable l'agencement de la croissance des seuls limbes.

Si on prend comme exemple la croissance du limbe $\mathrm{n}^{\circ} 6$ (annexe), on voit que la croissance de cet organe s'effectue par étapes. Depuis son initiation, observée au stade foliaire 1,0, le jeune limbe subit une étape de croissance dite modérée jusqu'au stade foliaire 4,0 , au moment où le limbe précédent $\left(\mathrm{n}^{\circ} 5\right)$ émerge. Cette étape couvre 3 thermochrones. Puis le jeune limbe aborde une étape de croissance rapide (le taux de croissance est multiplié par 3) pendant un thermochrone ; survient alors l'émergence. La croissance, visible à ce moment, est de type amorti et s'achève en un thermochrone. Cette organisation par étapes se déplace d'un phytomère à l'autre de manière acropète. En effet, lorsque le limbe $n^{\circ} 6$ émerge, c'est au tour du limbe suivant $\left(n^{\circ} 7\right)$ d'entrer en croissance rapide, pendant un thermochrone également, etc..., jusqu'à l'émergence du limbe $n^{\circ} 9$, le dernier limbe (fig. 1 et 2 ).

Ce principe de croissance par étapes (modérée, rapide, amortie) intervient pour l'ensemble des organes, mais selon des modalités propres à chacun d'eux (fig. 2 à 5 et tabl. 1). L'observation de la mise en place du système vasculaire permet d'en préciser la logique.

\section{B. Mise en place du système vasculaire}

L'observation des coupes fines longitudinales, colorées au carmin, permet de distinguer 2 ensembles dans la plante, représentée schématiquement figure 6 .

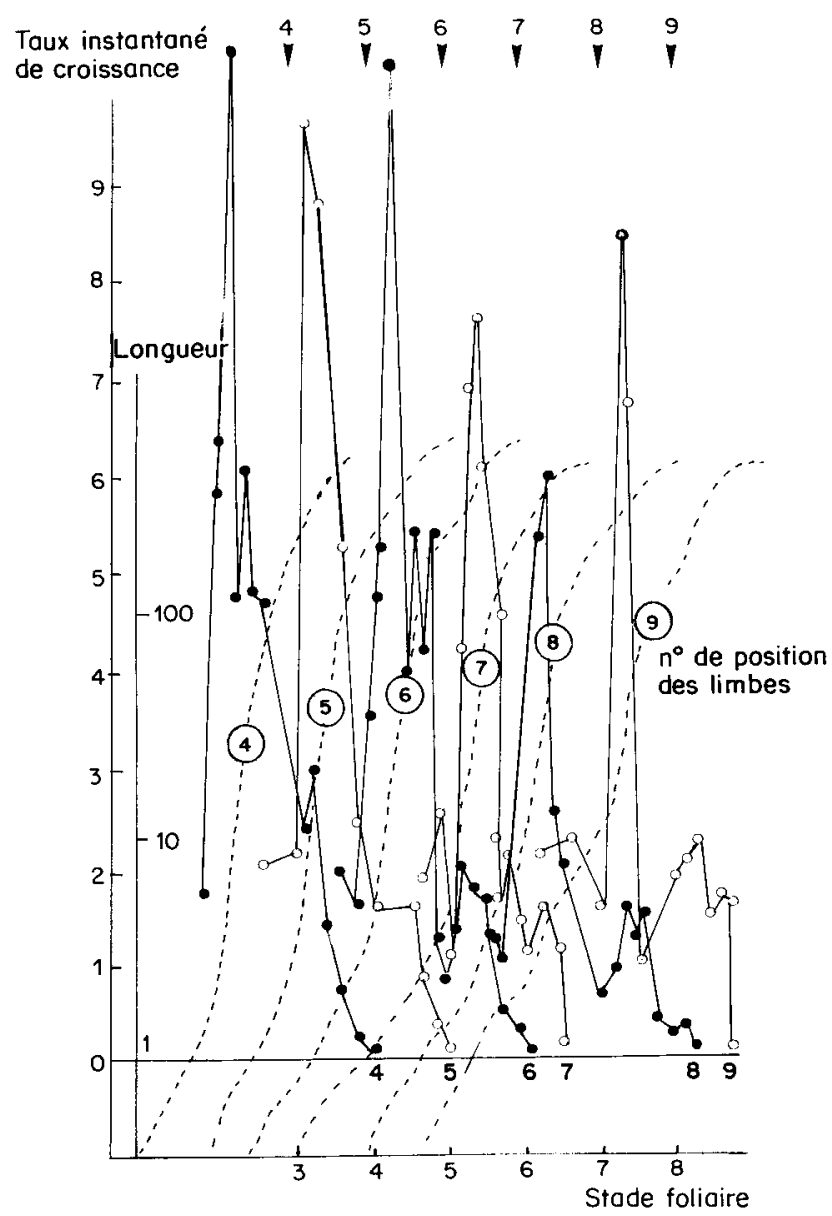

Figure 1

Evolution, en fonction du temps biologique, de la croissance des limbes 4 à $9 ;. . .$. : croissance en longueur des limbes $(\mathrm{mm})$; - $-0 \_$, taux de croissance en $\mathrm{mm} . \mathrm{mm}^{-1}$ par thermochrone. $\boldsymbol{\nabla}$ : émergence de la feuille.

Growth of laminae 4 to 9 in relation to biological time. .... : growth in length $(\mathrm{mm}) ; \bullet-\bullet, 0-0$ : relative growth rate (mm. $\mathrm{mm}^{-1}$ by thermochrone). $\mathbf{\nabla}$ : leaf appearance.

En partant du dôme terminal, on distingue un $1^{\text {er }}$ ensemble, noté $\mu$, où aucun signe de vascularisation n'est visible dans le secteur nodal des phytomères néoformés. Puis un ensemble de 4 positions, notées successivement $\alpha, \beta, \gamma, \delta$, présentant les caractéristiques suivantes :

- $\alpha$, correspondant au limbe enroulé, en croissance rapide, non encore visible. En $\alpha$ débute la provascularisation du nœud,

- $\beta$, correspondant au limbe émergeant, en expansion visible (croissance amortie). Le plexus nodal, constitué de fins éléments entremêlés, se met en place,

- $\gamma$, le limbe est pleinement sorti, le plexus nodal est « envahi », le nœud atteint sa taille définitive. On notera que la position $\gamma$ correspond au nombre entier du stade foliaire, la position $\beta$ au nombre en décimal,

$-\delta$, cette position correspond à la feuille adulte ; le nœud, au sens agronomique du terme, apparaît par épaississement du pulvinus.

Le phytomère $\delta$ occupe une position charnière entre l'ensemble $\alpha, \beta, \gamma$ situé au-dessus - oú, comme nous allons le voir, se déroule la croissance effective des 


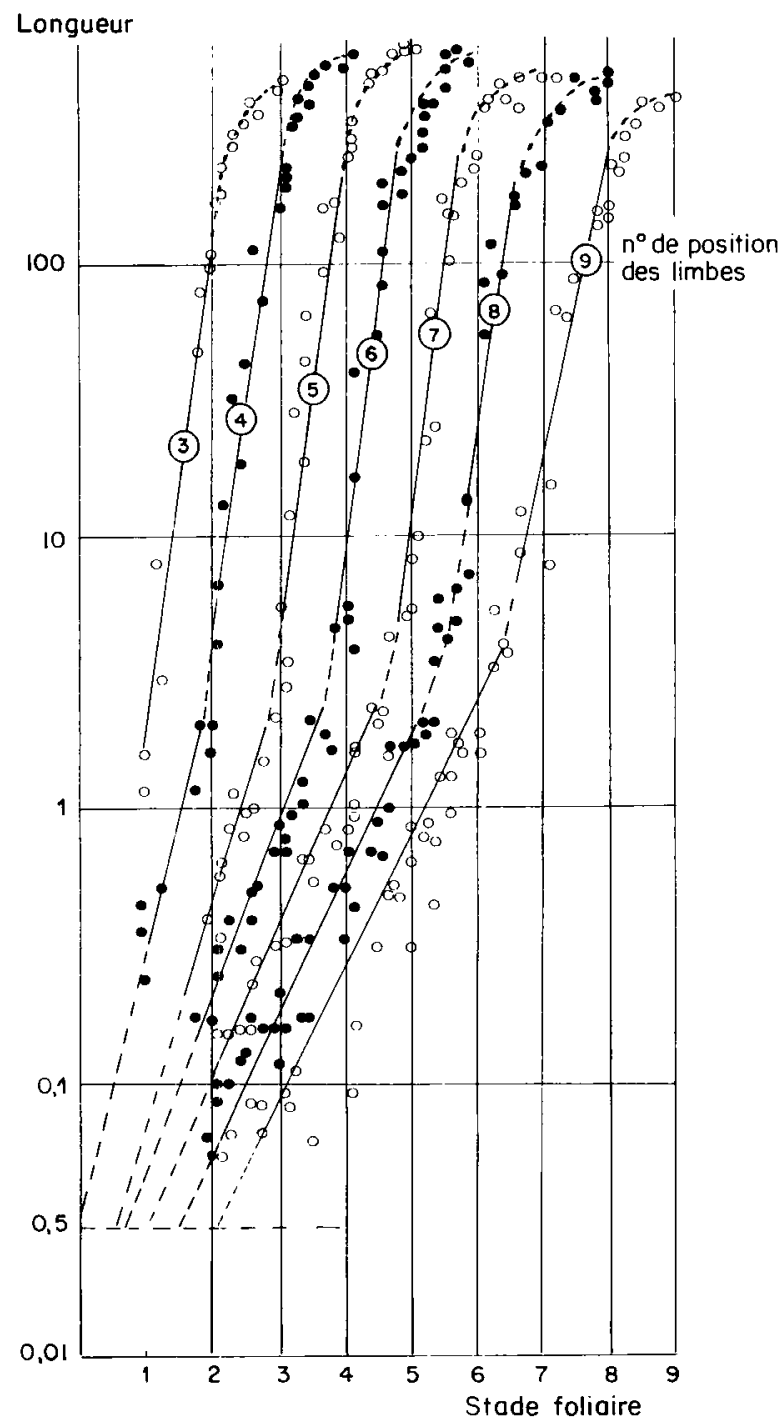

Figure 2

Croissance en longueur (mm) des limbes 3 à 9 en fonction du temps biologique.

Elongation ( $\mathrm{mm}$ ) of laminae 3 to 9 in relation to biological time.

organes - et l'ensemble situé en dessous, celui des phytomères matures et sénescents.

Cette description succincte matérialise ce que l'on voit à la loupe binoculaire à partir d'une coupe fine à main levée ; elle sera complétée par les observations de coupes sériées rapportées par PATRICK (1972a et $b$ ) et commentées dans la discussion.

\section{Coordination des croissances intra- et inter- phytomères}

Si on étudie simultanément l'ensemble des courbes de croissance, en les superposant, comme le montre par exemple la figure 7 , et en les rapprochant de l'évolution de la vascularisation, on obtient une schématisation de la croissance sous-apicale, d'une remarquable simplicité (fig. 8).

- L'ensemble $\mu$ est celui de tous les phytomères à croissance modérée. Ces phytomères, empilés du dôme terminal à la position $\alpha$ exclue, sont en "attente" ; aucun signe de vascularisation n'est visible dans le secteur nodal de ces phytomères.

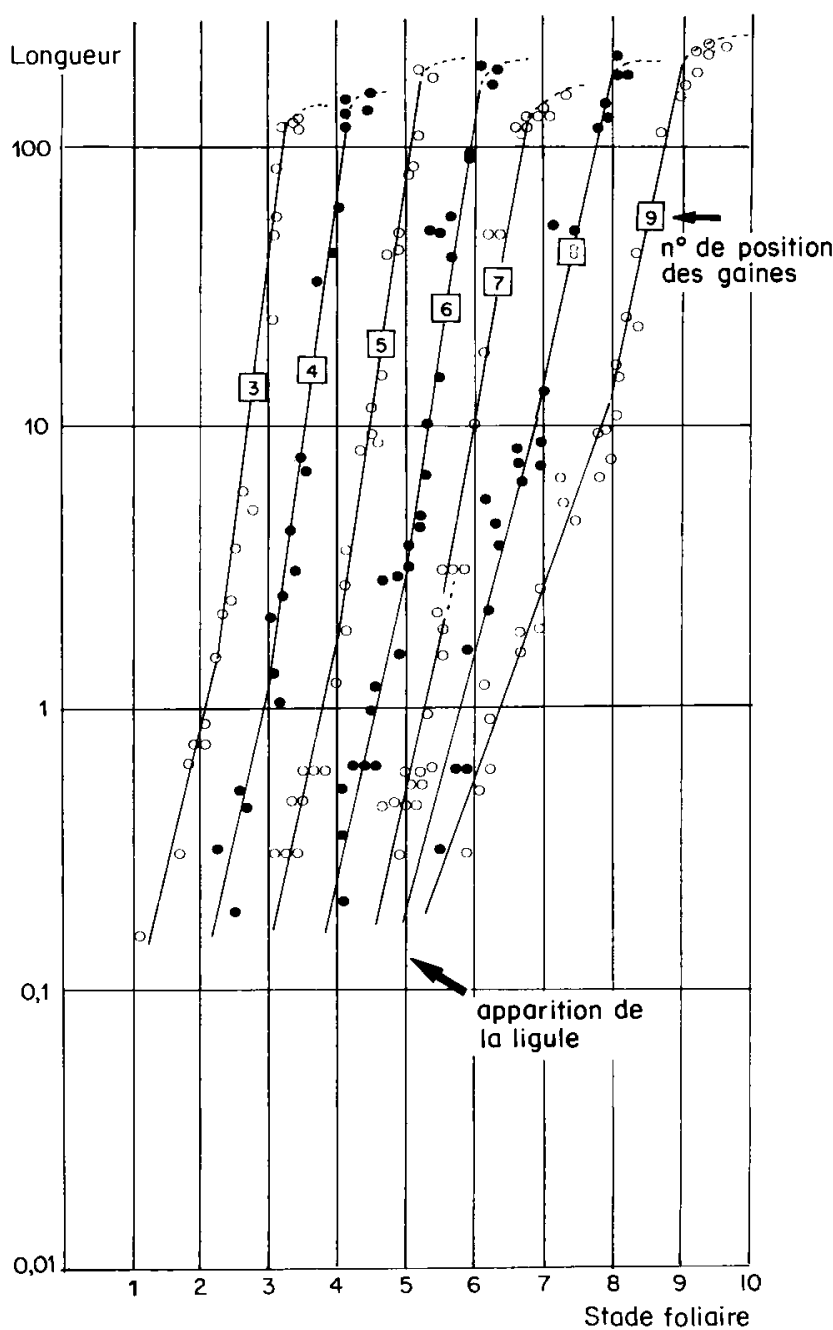

Figure 3

Croissance en longueur ( $\mathrm{mm}$ ) des gaines 3 à 9 en fonction du temps biologique.

Elongation ( $\mathrm{mm}$ ) of sheaths 3 to 9 in relation to biological time.

- L'ensemble des 4 positions $\alpha, \beta, \gamma, \delta$ joue le rôle d'une véritable "trappe " de croissance: en effet, lorsque le phytomère $\alpha+1$, dont tous les organes étaient jusque-là en croissance modérée, arrive en position $\alpha$, le limbe, la gaine dont la croissance semblait inhibée et l'entre-nœud, s'il existe, entrent brutalement dans une phase de croissance rapide. La croissance rapide se poursuit dans la gaine et l'entre-nœud à l'étape suivante (position $\beta$ ) ; ces deux organes sont encore cachés par la gaine de la feuille précédente (position $\gamma$ ) alors que le limbe, maintenant visible, est en croissance amortie. Puis, à l'étape suivante (position $\gamma$ ), seul l'entre-nœud subira éventuellement une phase de croissance rapide. En $\alpha$, $\beta, \gamma$ le nœud se vascularise, en $\delta$ il se lignifie, il n'y aura plus de croissance rapide. Ce système de 4 positions $\alpha, \beta, \gamma, \delta$ fonctionne pas à pas jusqu'à l'épiaison, chaque position étant occupée par un phytomère, pendant une étape élémentaire de durée égale à un thermochrone. A l'émergence de la dernière feuille, le dernier nœud se trouvant donc en position $\beta$, c'est au tour du col de l'épi et de l'inflorescence situés au-dessus du dernier nœud d'entrer dans la " trappe " de croissance en position $\alpha$ (fig. 9). On 


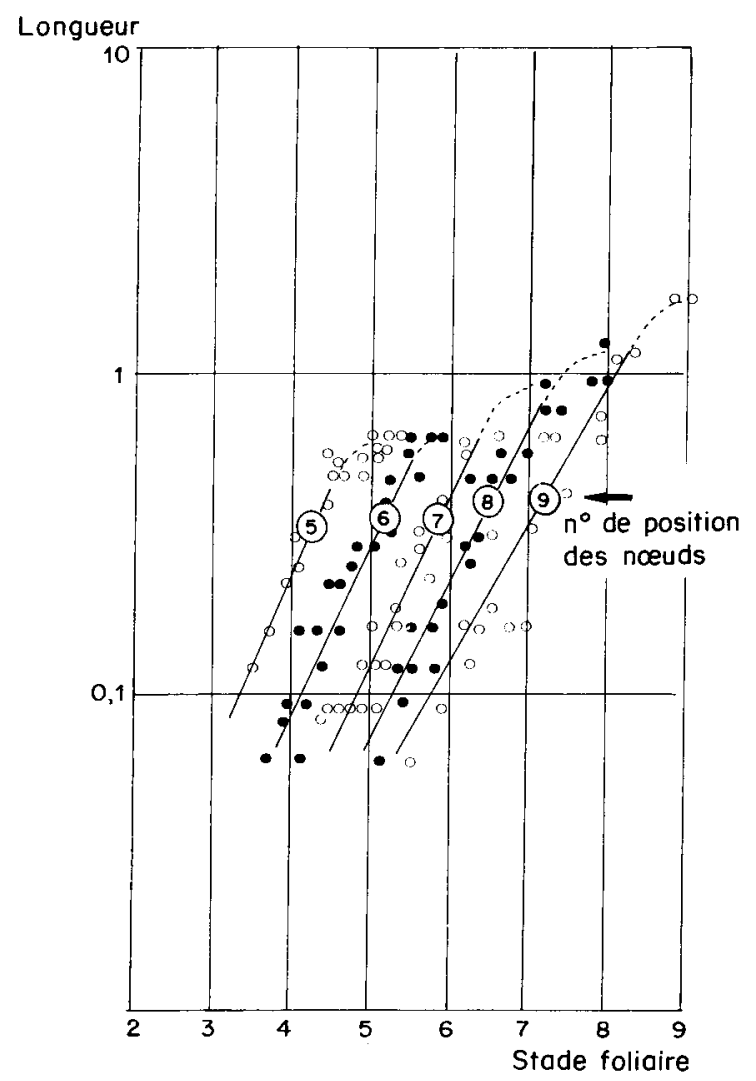

Figure 4

Croissance en longueur (mm) des nouds 5 à 9 en fonction du temps biologique.

Elongation ( $\mathrm{mm}$ ) of nodes 5 to 9 in relation to biological time.

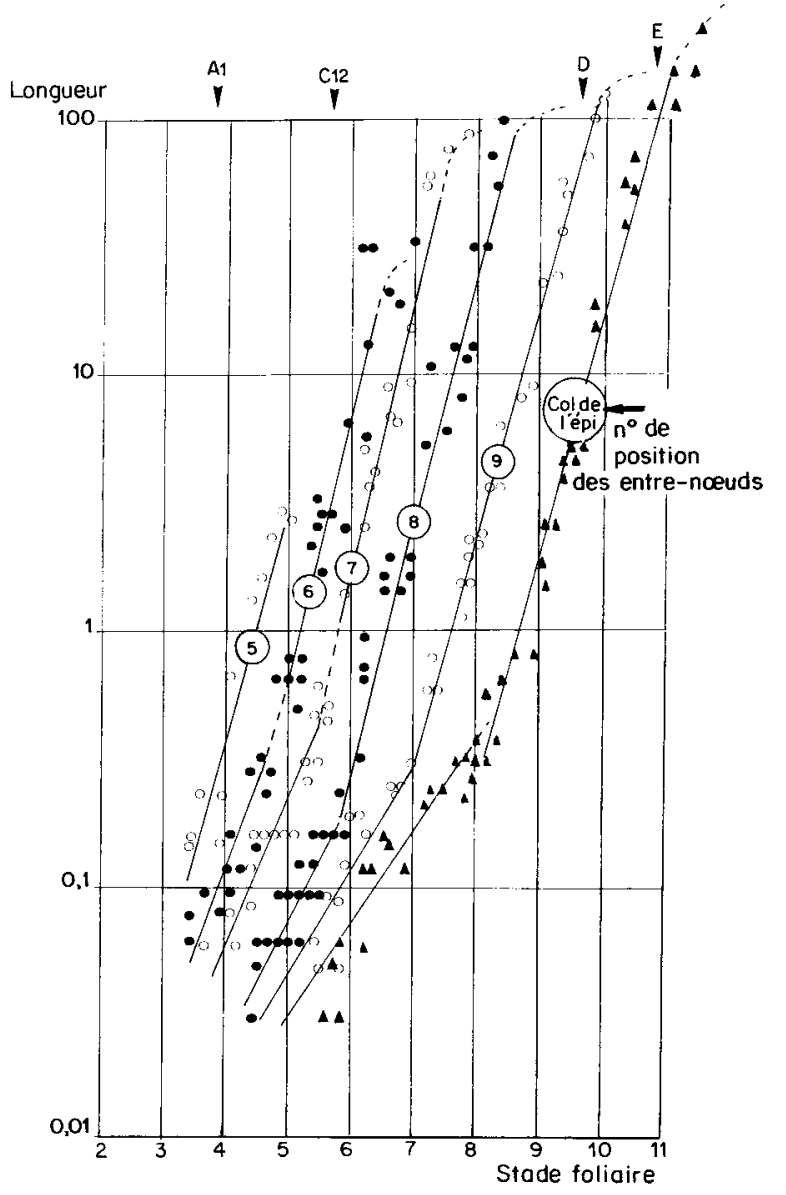

Figure 5

Croissance en longueur (mm) des entre-nœuds 5 à 9 et du col de l'épi ; A1. ...D. (voir MALVOISIN 1984, cf. annexe).

Elongation (mm) of internodes 5 to 9 and of the neck in relation to biological time ; A1. ... D. (see MALVOISIN 1984, cf. annex).

TABLEAU 1

Taux moyens de croissance des différents organes végétatifs et de l'inflorescence (en $\mathrm{mm} . \mathrm{mm}^{-1}$ par thermochrone).

Relative growth rate of the different vegetative organs and of the inflorescence ( $\mathrm{mm} . \mathrm{mm}^{-1}$ per thermochrone).

\begin{tabular}{|c|c|c|c|c|c|c|c|}
\hline \multirow{2}{*}{$\mathrm{n}^{\circ}$ de position } & \multirow{2}{*}{$\begin{array}{c}\text { Nœud } \\
\text { Modérée }\end{array}$} & \multicolumn{2}{|c|}{ Entre-nœud } & \multicolumn{2}{|c|}{ Gaine } & \multicolumn{2}{|c|}{ Limbe } \\
\hline & & Modérée & Rapide & $\begin{array}{l}\text { Rapide } \\
1^{\text {re }} \text { phase }\end{array}$ & $\begin{array}{c}\text { Rapide } \\
2^{\mathrm{e}} \text { phase }\end{array}$ & Modérée & Rapide \\
\hline 3 & & & & 2,16 & 4,44 & & 4,23 \\
\hline 4 & & & & 2,49 & 4,28 & 2,20 & 3,91 \\
\hline 5 & 1,26 & & 2,11 & 2,58 & 3,81 & 1,76 & 4,03 \\
\hline 6 & 1,22 & 1,43 & 2,37 & 2,47 & 3,72 & 1,42 & 4,18 \\
\hline 7 & 1,22 & 1,30 & 2,32 & 2,53 & 3,10 & 1,27 & 4,17 \\
\hline 8 & 1,13 & 1,05 & 2,18 & 2,43 & 2,61 & 1,15 & 3,78 \\
\hline 9 & 0,99 & 0,95 & 2,10 & 1,55 & 2,74 & 1,13 & 2,56 \\
\hline $\begin{array}{l}\text { col } \\
\text { épi }\end{array}$ & & 0,83 & 2,13 & & & & \\
\hline inflorescence & & $\begin{array}{c}\text { modérée } \\
0,57\end{array}$ & $\begin{array}{c}\text { semi-rapide } \\
1,44\end{array}$ & & & & \\
\hline
\end{tabular}




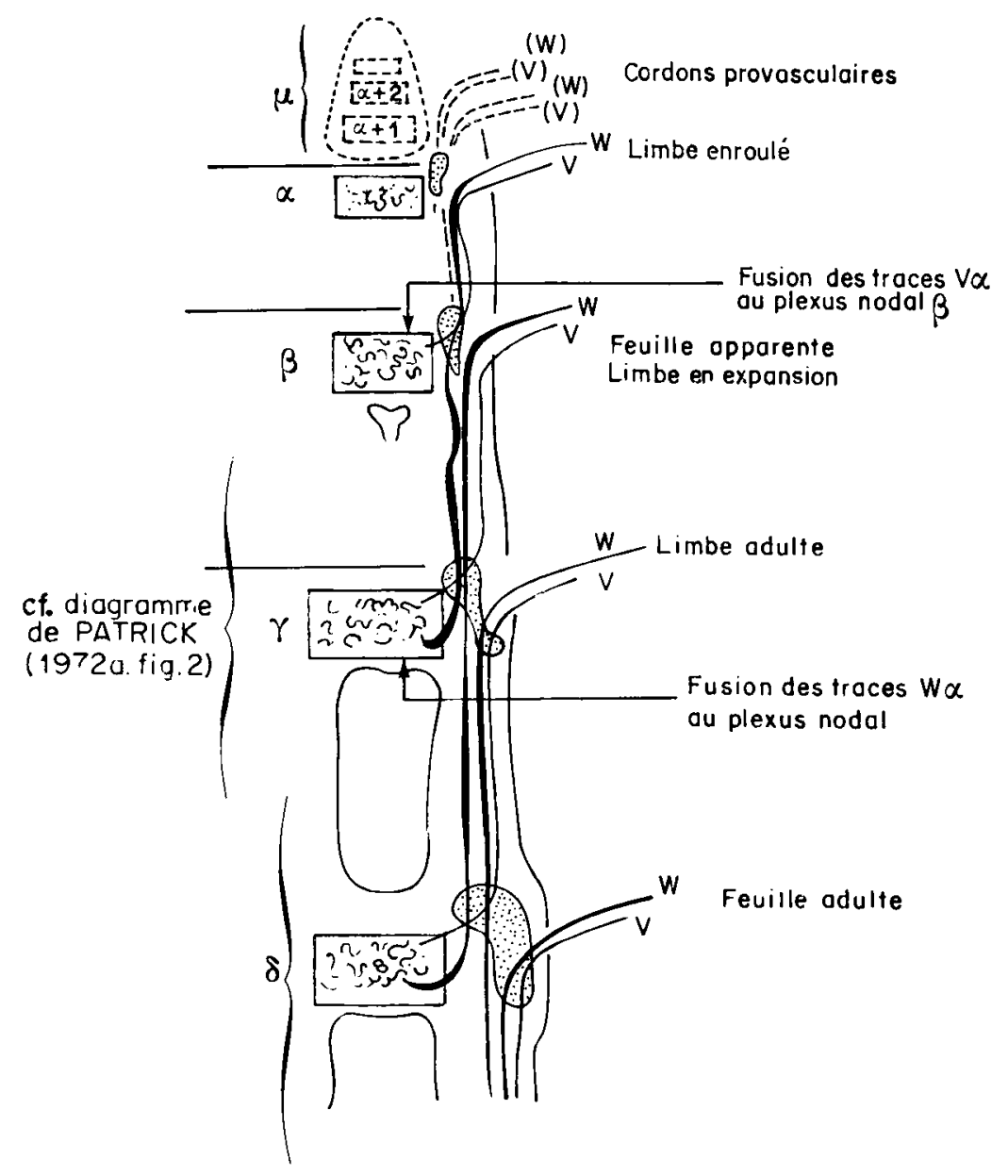

Figure 6

Mise en place de ia vascularisation de la tige; modifié, d'après PATRICK (1972). V, W voir figure 10.
Vascularization pattern of the wheat stem; modified data from PATRICK (1972). $V, W$, see figure 10. note à ce propos que l'observation de l'émergence de la dernière feuille peut constituer un repère intéressant puisqu'elle permet de scinder la période florale en une $1^{\text {re }}$ partie, caractérisée par une croissance modérée de l'épi et du col, et une $2^{\mathrm{e}}$ partie, marquée par une croissance rapide de ces mêmes organes. A l'issue de l'émergence de la dernière feuille, apparaît la gaine de cette feuille, le dernier nœud se trouve alors en position $\gamma$. C'est au cours de cette étape que se produit la méiose dans l'épi (fig. 9), la ligule de la dernière feuille étant dégagée de quelques $\mathrm{cm}$. Puis survient l'épiaison (fin de la position $\gamma \mathrm{du}$ col de l'épi) et la floraison (fin de l'expansion du col, en position $\delta$ ). Ainsi s'achève la $1^{\text {re }}$ partie du cycle de morphogenèse, l'activité de croissance intéressant alors le développement de l'embryon et du grain, il n'y a plus de croissance dans les organes végétatifs de la plante.

\section{Les deux domaines de la plante}

L'examen du fonctionnement de cette activité de croissance soulève une question importante. En effet, si on se rappelle que la position $\gamma$ correspond au nombre entier du stade foliaire (et la position $\beta$ au nombre décimal), on constate que la gaine $\gamma$, en croissance amortie, enveloppe la gaine et une partie du limbe $\beta$ émergeant, respectivement en croissance rapide et amortie, ainsi que le limbe $\alpha$, enroulé et non visible, en croissance rapide. On peut alors supposer que l'émergence du limbe $\alpha$ et de la gaine $\beta$ hors du tube formé par la gaine $\gamma$ constitue l'événement censeur de la translation périodique de la " trappe » de croissance $\alpha, \beta, \gamma$. Deux domaines de la plante se distinguent :

1) le domaine de la morphogenèse, domaine enveloppé par la gaine $\gamma$, qui est le domaine des territoires capables de croissance. C'est un domaine protégé qui se trouve dans une situation d'humidité optimale, où la lumière reçue est une lumière filtrée par les couches chlorophylliennes (à dominante rouge, rouge sombre ?). Ce domaine contraste avec :

2) le domaine apparent, domaine de la photosynthèse. C'est le domaine de la plante qui se trouve au contact de l'atmosphère, dont les territoires sont saisis par une sclérification brutale qui arrête la croissance. Tout se passe comme si l'arrivée « à l'atmosphère " du limbe $\alpha$ et de la gaine $\beta$, entraînait une translation automatique du système. 


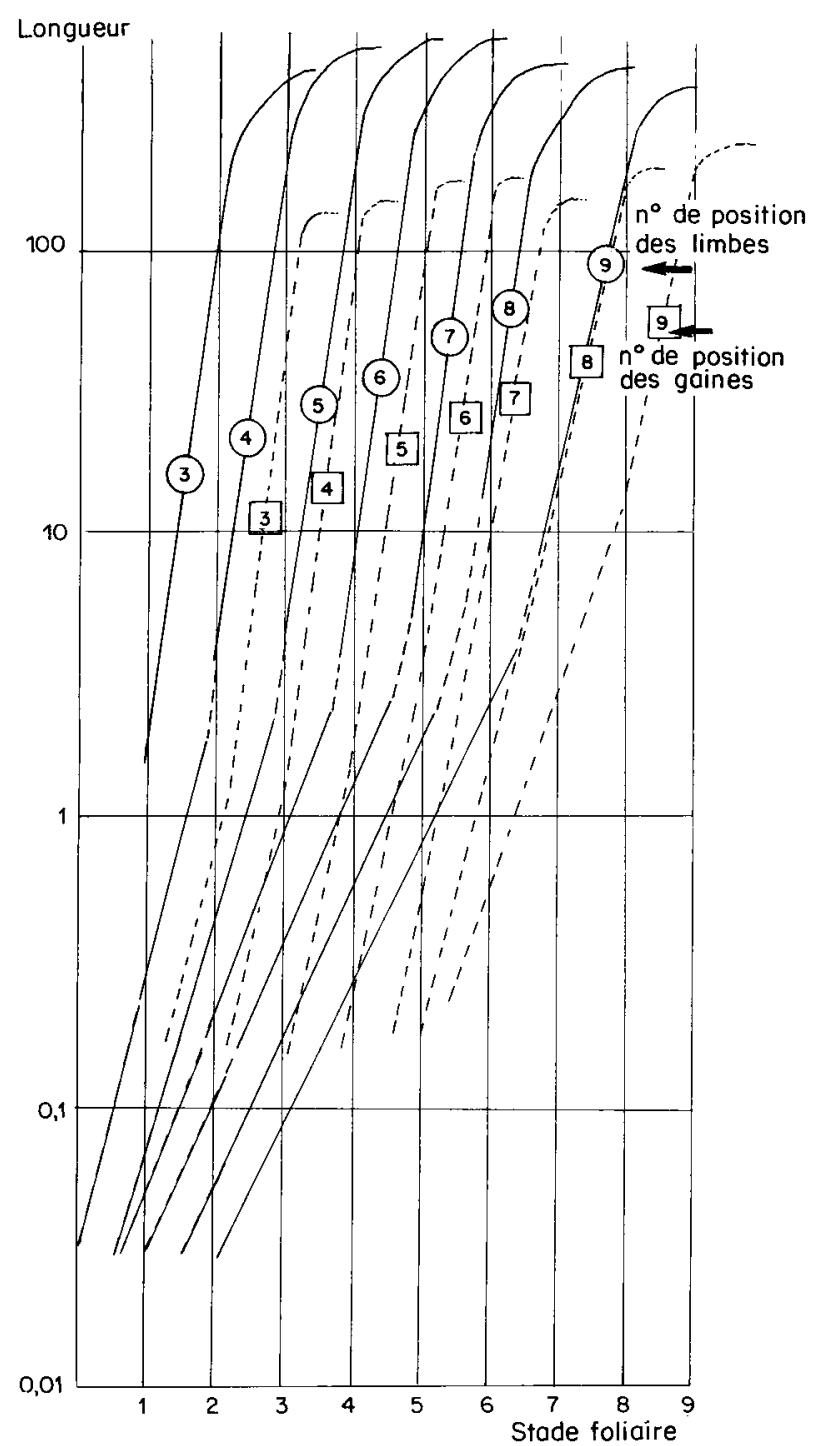

Figure 7

Croissance comparée des limbes (trait plein) et des gaines (trait pointillé), d'après les ajustements présentés figures 2 et 3.

Comparative growth curves of laminae (continuous line) and sheaths (dotted line); data from figures 2 and 3.

Figure 9

Croissance comparée de l'inflorescence ( $\mathbf{\square})$, des nœuds $(0$, $\bullet$ ) et du col de l'épi (४) ; longueurs en mm. ; Al, .., E, voir MALVOISIN 1984, cf. annexe.

Comparative growth curves of the inflorescence ( $\mathbf{\square})$, of the nodes $(0, \bullet)$ and of the neck (A); $A 1, \ldots$, E, see MALVOISIN 1984, cf. annex.

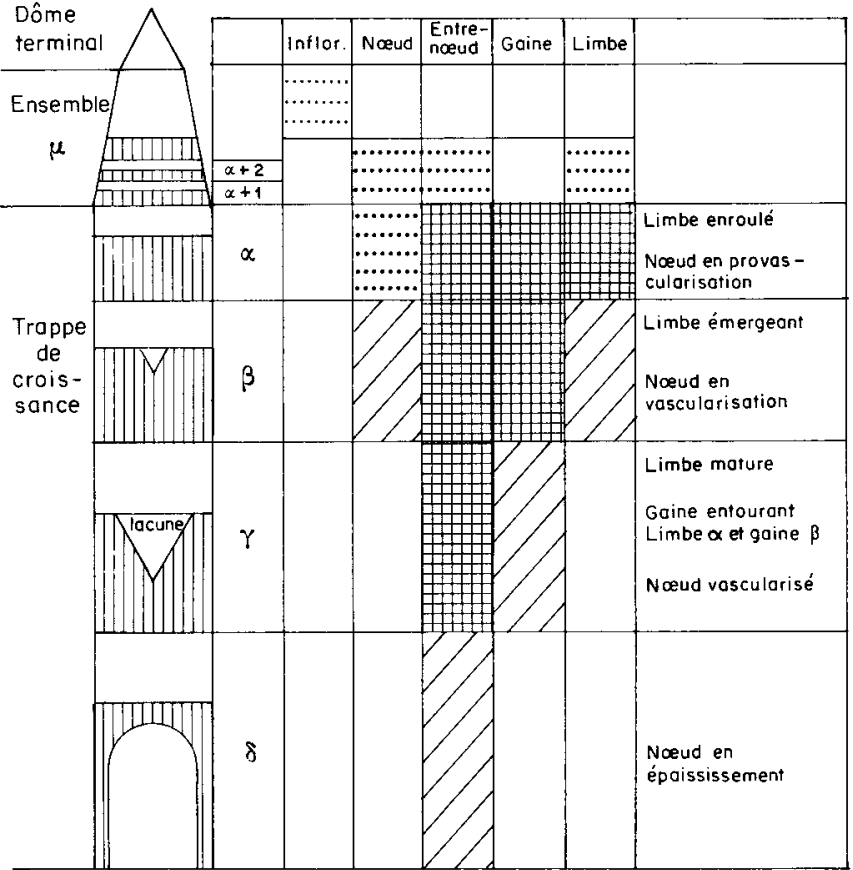

Figure 8

Coordination de la croissance sous-apicale des composants de la zone de croissance (période transitoire).

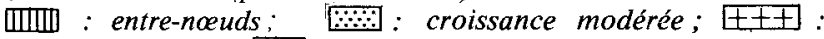
croissance rapide ; $\because$ :croissance amortie $; \mu . \ldots \delta$ (voir fig. 6).

The coordinated growth pattern of the component parts of the growing point (transition period).

血血 : internodes; rapid g.r. ; $\mathbb{Z}$ : declining g.r. ; $\mu \ldots \delta$ (see fig. 6).

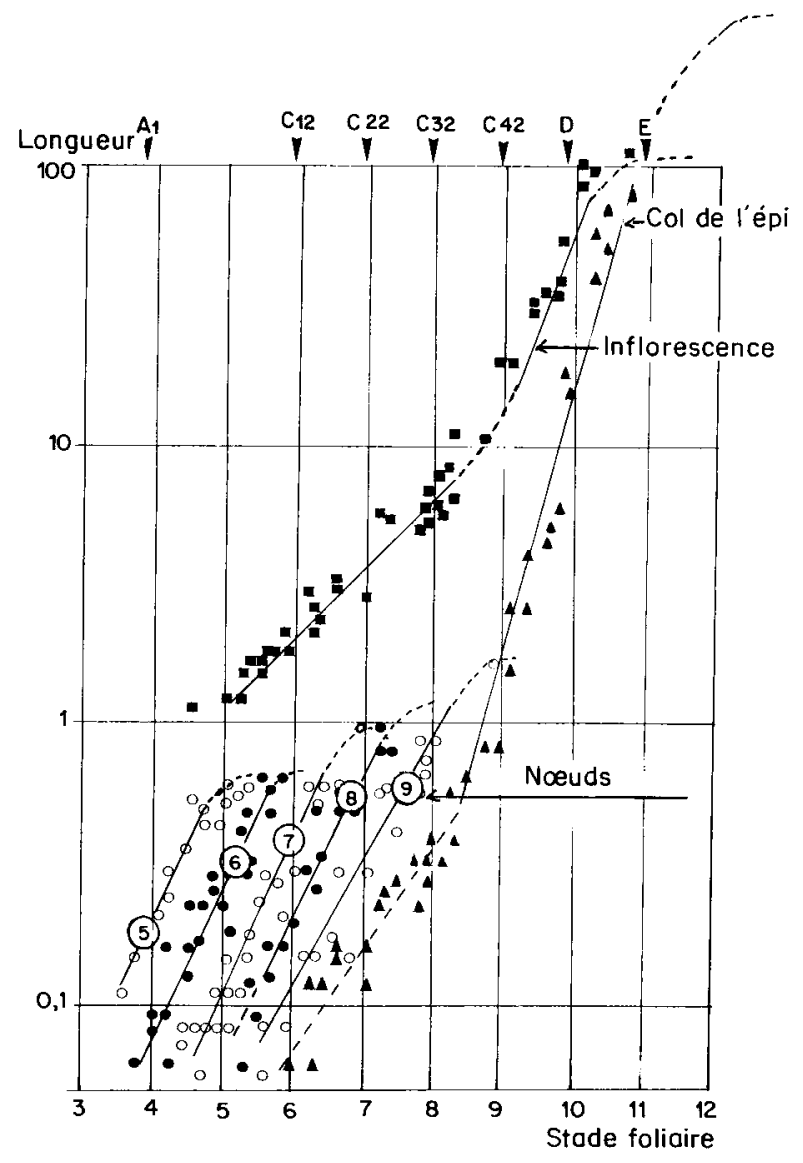




\section{DISCUSSION}

Williams (1975), pour l'étude de la croissance de la feuille, et PATRICK $1972 a$ et $b$ ) pour celle de la mise en place du système vasculaire, apportent des précisions qui complètent notre analyse.

Williams a étudié en détail la croissance de la feuille $\mathrm{n}^{\circ} 4$ (limbe et gaine) d'un blé de printemps placé dans des conditions assez semblables aux nôtres. Outre la croissance en longueur, WILLIAMS a étudié, par des microanalyses, la croissance en matière sèche (en la ventilant en protéines, parois cellulaires, $A R N$ et $A D N)$, l'évolution estimée du volume cellulaire et du nombre de cellules. WILLIAMS remarque bien la brusque augmentation du taux de croissance (relative growth rate) au début de ce que nous avons convenu d'appeler l'étape $\alpha$ de la croissance du limbe ; il note aussi une forte intensification de la production de parois cellulaires pendant cette même période, confirmant ainsi, comme nous l'avons décrit de manière imagée, l'entrée dans la "trappe " de croissance effective du phytomère $\alpha+1$ jusque-là en croissance modérée. L'examen des données de WILliams permet d'attribuer un caractère euméristématique à l'ensemble des phytomères "en attente" (ensemble $\mu$ ), les paramètres de croissance cellulaire restant stables pendant cette période; par contre, l'arrivée, dans la " trappe » $\alpha, \beta, \gamma, \delta$, des phytomères entraîne ces territoires dans une phase de croissance cellulaire par élongation intense (croissance effective).

La participation du système vasculaire au déroulement de la morphogenèse doit être précisée; PATRICK $(1972 a$ et $b)$ a réalisé, à la suite de SHARMAN (1967), une étude détaillée de la mise en place du système vasculaire du blé, fondée sur l'examen de coupes transversales sériées. Les observations rapportées par PATRICK sont abondantes et complexes; VINCENT (1982) en propose des éléments d'interprétation qui s'avèrent particulièrement éclairants : d'après VINCENT, on doit d'abord distinguer 2 ensembles de faisceaux, l'ensemble $V$ et l'ensemble $W$ (fig. 10). Cette distinction, importante quant à ses conséquences, n'apparaît pas dans le travail de PATRICK : VINCENT observant une série de coupes transversales d'un primordium foliaire (WILliams, 1975) remarque qu'un primordium est constitué par 8 colonies dorsales primaires, issues chacune d'une cellule, et par

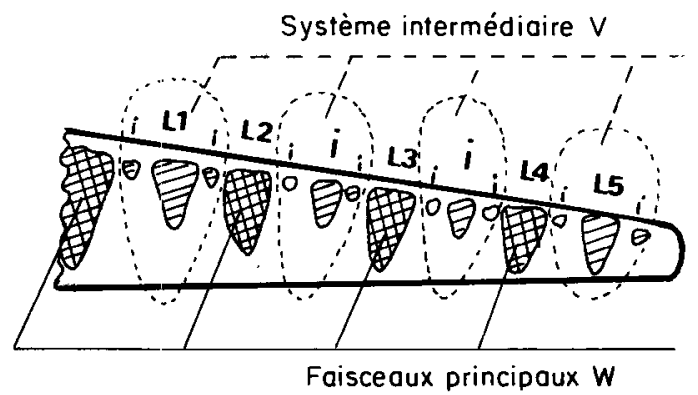

Figure 10

Schéma d'une coupe transversale d'un demi-limbe de blé; schéma modifié, d'après PATRICK (1972 a, fig. I).

Diagram of the cross section of half a wheat lamina ; figure modified after PATRICK (1972a, fig. l).
7 colonies ventrales intercalées; les axes primaires de provascularisation sont situés exactement aux frontières de ces colonies cellulaires : cet agencement conditionne celui du système vasculaire en 7 faisceaux majeurs, appelés $W$ dont les traces se prolongent de 2 positions jusqu'au nœud inférieur, et en 8 systèmes intermédiaires, nommés $V$, dont les traces se prolongent à la périphérie du nœud inférieur de 1 position (fig. 6). Chaque système $V$ est lui-même formé d'un faisceau majeur flanqué de 2 faisceaux satellites de formation différée (fig. 10).

En interprétant les données de PATRICK, il se confirme que la provascularisation du nœud débute en position $\alpha$; aucun signe de provascularisation n'est noté dans le méristème médullaire des phytomères composant l'ensemble $\mu$. A la périphérie du nœud $\alpha$, PATRICK constate une disparition des terminaisons des éléments $V \alpha+1$, alors qu'un peu plus bas des foyers procambiaux s'installent à l'aisselle interne des traces $V \alpha$. Ces. foyers engendrent de nouvelles structures adossées aux traces $W \alpha+1$, donc au nombre de 7 par nœud: ce sont les "piliers de transfert" (basal strands), constitués d'un réseau de fins éléments de phloème associés à des cellules de transfert. Ces cellules, à parois invaginées en de multiples plis, peuvent être considérées comme des modules d'échanges entre les domaine externe (xylème et parois) et interne (phloème). Le rôle de ces cellules, pendant cette phase de la morphogenèse, reste à préciser. Simultanément à cette activité périphérique, une activité indépendante s'initie à l'intérieur du nœud $\alpha$ et se propage à partir de sa base. Elle engendre le plexus nodal. Ce système plexus-piliers de transfert poursuit son expansion tout au long de la " trappe " de croissance $\alpha, \beta, \gamma, \delta$ tout en se connectant aux traces foliaires de manière très précise (VINCENT, 1982).

La confrontation de la mise en place du système vasculaire avec l'organisation de la croissance sous apicale, telle que nous l'avons décrite, apporte des éléments pour construire un modèle de fonctionnement intégré de la morphogenèse. VINCENT (1982) en propose un qui verrait dans le fonctionnement de la trappe $\alpha, \beta, \gamma, \delta$ l'intervention d'un flux distribué par le jeune système vasculaire en installation, en considérant les piliers $\alpha, \beta, \gamma$, voire $\delta$, comme générateurs de flux et en supposant l'existence de transfert de charges entre les systèmes $\mathrm{V}$ et $\mathrm{W}$ aux sommets du limbe $\alpha$ et de la gaine $\beta$. Cette " conductibilité », propre aux sommets, cesserait à l'émergence de ces organes hors de la gaine $\gamma$ ce qui entraînerait la translation du système.

Enfin, pour compléter cette discussion, il nous faut rappeler les résultats dégagés dans une précédente étude (MALVOISIN, 1984) concernant l'existence d'une relation entre le fonctionnement de la croissance sous-apicale (c'est-à-dire le fonctionnement de la trappe $\alpha, \beta, \gamma$ et $\delta$ ) et l'activité organogène apicale :

- Création de 2 initiums par thermochrone pendant la période juvénile ;

- Accélération du rythme d'initiation au moment de l'arrivée en $\alpha$ d'un phytomère critique auquel sera associé le $1^{\text {er }}$ entre-nœud (stade A1), pendant la période de transition;

- Retour à un rythme régulier avec la réalisation d'un pas floral (annexe 1) par thermochrone (fig. 9) 
MALVOISIN 1984, cf. annexe pendant la période florale. L'idée d'une activité autonome de l'apex, ou asservie par la zone sous-apicale par l'intermédiaire du système vasculaire et provasculaire, reste une question qui divise les morphogénéticiens (HALPERIN, 1978). Peut-être faut-il s'orienter vers une conception intégrant à la fois un rôle organisateur de l'apex, au sens de NOZERAN et al. (1971) et une fonction d'asservissement de la zone sous-apicale sur les méristèmes liée aux fluctuations de flux énergétique qui atteignent la zone apicale (CNRS, 1979). Ces fluctuations pourraient être créées par la translation nœud après nœud de la trappe de croissance $\alpha$, $\beta, \gamma, \delta$.

\section{CONCLUSION}

Les résultats sont rassemblés dans un schéma présenté figure 11. Ce schéma de fonctionnement de la plante - fonctionnement pris au sens cybernétique du terme - vise à délimiter les secteurs en activité et leurs relations. Deux pôles d'activité apparaissent :

- Le dôme terminal, siège de l'initiation des phytomères, relayé ensuite par la population des bourgeons d'épillet qui constitue le futur épi.

- Un ensemble de 4 phytomères successifs $\alpha, \beta, \gamma$ et $\delta$ où se déroulent les processus de croissance effective, $\gamma$ représente le nombre entier du stade foliaire, $\beta$ correspond à la position de la feuille en expansion, $\alpha$ à celle de la feuille enroulée, non visible. Entre ces 2 pôles d'activité se constitue une zone de phytomères empilés, en attente (zone $\mu$ ) ; cette zone verra ses caractéristiques évoluer pendant la période de transition: selon les conditions de milieu (interaction photopériode-vernalisation), le nombre final de phytomères végétatifs (nombre final de feuilles) sera plus ou moins important ce qui entraînera une durée plus ou moins longue du parcours. L'activité de croissance, que nous avons caractérisée par le fonctionnement de la «trappe » $\alpha, \beta, \gamma$ et $\delta$ se déṕlace, quant à elle, de manière acropète, remontant régulièrement, par étapes successives, nœud après nœud, la plante. La durée d'une étape élémentaire est donnée par le thermochrone. Cette activité semble ordonner l'ensemble de la morphogenèse d'après les hypothèses formulées dans la discussion.

D'un point de vue pratique et méthodologique, sachant que la position $\gamma$ correspond au nombre entier du stade foliaire, nous sommes en mesure de déduire immédiatement les états de croissance de tous les organes, à chaque instant. De plus, en connaissant le thermochrone, les stades foliaires correspondant au début et à la fin de la transtion et le nombre final de feuilles, il est possible de décrire entièrement et avec précision le déroulement de l'organogenèse et de la croissance du maître-brin, du semis à l'épiaison, dans cette $1^{\text {re }}$ partie du cycle de morphogenèse, au terme de laquelle se déterminera une potentialité de la plante qui s'affichera à la récolte.

Reçu le 26 août 1982. Accepté le 5 mars 1984.

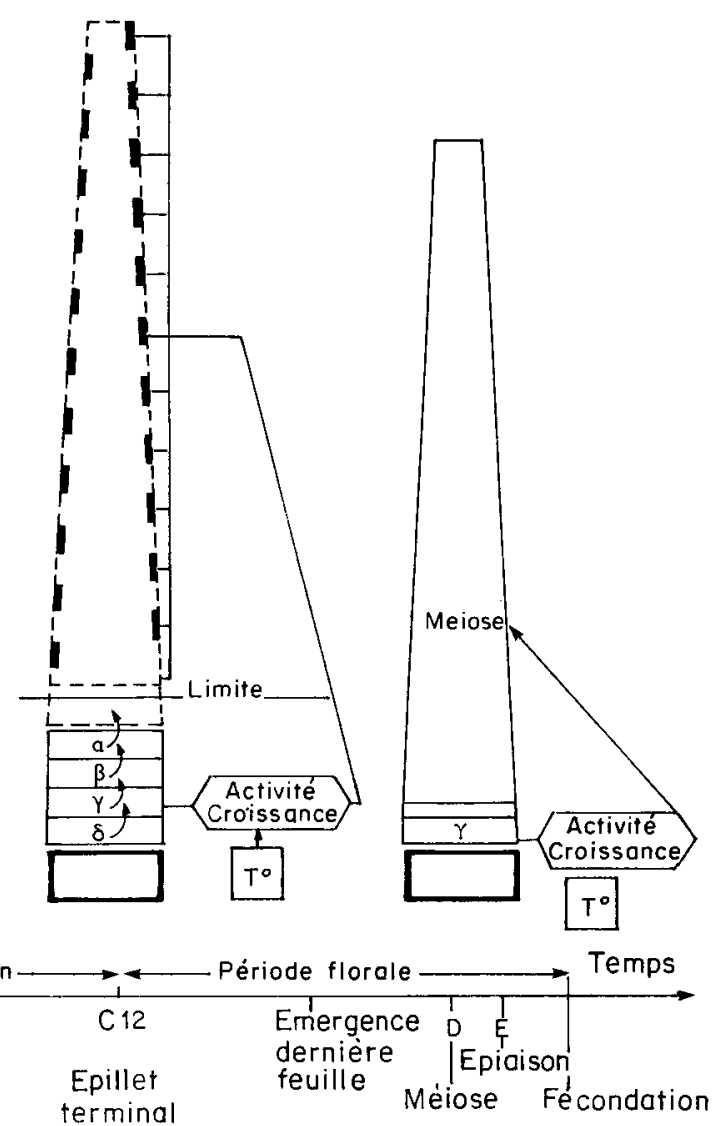

Figure 11

Modèle du fonctionnement intégré du maître-brin du blé, du semis à l'épiaison. $\mathbf{A}$ : dôme terminal; $\mathbf{\square}$ : bourgeons d'épillets et de fleurs; $;:$ ensemble $\mu$ des phytomères « en attente»; $\square$ : ensemble $\alpha, \beta, \gamma, \delta$ ou " trappe » de croissance effective, voir figure 8 ; $\square$ :ensemble des phytomères matures $; A 1, \ldots E$, voir MAL VOISIN 1984, cf. annexe.
Model of an integrated growth pattern for the wheat culm from seeding to heading stages. $\mathbf{\Delta}$ : shoot apical meristem; : spikelet and floret bud meristems, $;:-j$ region; $\square: \alpha, \beta, \gamma, \delta$ region, see figure $8 ; \square$ : mature phytomers ; $A 1, \ldots E$, see MALVOISIN 1984, cf. annex. 


\section{REMERCIEMENTS}

Ce travail a été réalisé à la Station d'Amélioration des Plantes de Dijon (I.N.R.A.) avec l'appui financier de l'Institut Technique des Céréales et des Fourrages (I.T.C.F.).

\section{ANNEXE}

Interprétation des courbes de croissance Growth curves analysis

Si pendant un intervalle de temps $\Delta t$ la longueur subit un accroissement $\Delta \mathrm{l}$, la vitesse de croissance est exprimée par le rapport $\frac{\mathrm{dl}}{\mathrm{dt}}$ (limite de $\frac{\Delta \mathrm{l}}{\Delta \mathrm{t}}$ quant $\Delta \mathrm{t} \rightarrow 0$ ) ; si on rapporte l'accroissement de longueur $\Delta \mathrm{l}$ par rapport à la longueur 1 , le rapport $\mathrm{b}=\frac{\mathrm{dl}}{\mathrm{ldt}}$ soit $\frac{\mathrm{d}(\log 1)}{\mathrm{dt}}$ (limite de $\frac{\Delta \log (1)}{\Delta \mathrm{t}}$ quant $\mathrm{t} \rightarrow 0$ ) exprime un taux de croissance (relative growth rate, Williams \& RiJVEN, 1965). En ajustant les points par une régression linéaire (fig. 12a), nous faisons l'hypothèse que le taux de croissance est constant pour l'étape considérée ; la pente de la régression donne la meilleure estimation du taux (taux de croissance moyen). L'ajustement a été réalisé pas à pas de manière empirique, en commençant par les premières données ; à l'amorce d'une rupture dans la disposition des points, visible sur le graphique, on n'a retenu que les points qui, intégrés dans le calcul de la régression, maintiennent un coefficient de détermination élevé, sans affecter la valeur de la pente de la droite. On a ainsi ajusté les phases de croissance "modérée " et "rapide », la disposition des points s'y prêtant particulièrement bien (fig. 12a et tabl. 2) ; par contre, la phase de croissance «amortie » qui présente une tendance curvilinéaire très marquée a échappé à ce type d'analyse.

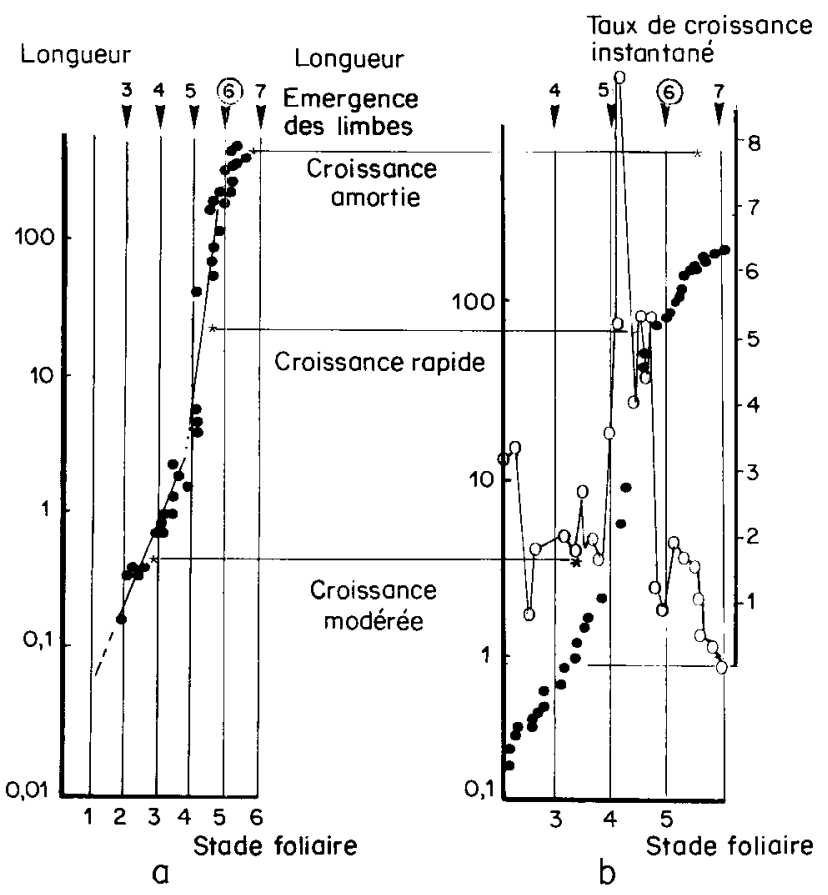

Figure 12

Ajustement des courbes de croissance en longueur. Cas du limbe $n^{\circ} 6$ « Cappelle $239 », 20^{\circ} \mathrm{C}, 16 \mathrm{~h} \bullet$ : longueur en $\mathrm{mm} ; 0:$ taux de croissance «instantané », en $\mathrm{mm} . \mathrm{mm}^{-1}$ par thermochrone. $\checkmark$ : émergence de la feuille.

Growth of the plant organs with time; case study of lamina $n^{\circ} 6$ of « Cappelle 239 » at $20^{\circ} \mathrm{C}$ and $16 \mathrm{~h}$ photoperiod. $\bullet$ : length $(\mathrm{mm}) ; 0$ : relative growth rate (mm. $\mathrm{mm}^{-1}$ thermochrone). $\boldsymbol{Y}$ : leaf appearance.
Tableau 2

Valeur des taux moyens de croissance: « Cappelle 239 », $20^{\circ} \mathrm{C}, 16 \mathrm{~h}$

Relative growth rates of lamina $n^{\circ} 6$; « Cappelle 239 », $20{ }^{\circ} \mathrm{C}, 16 \mathrm{~h}$

\begin{tabular}{lcc}
\hline \hline & $\begin{array}{c}\text { Phase } \\
\text { de croissance } \\
\text { modérée }\end{array}$ & $\begin{array}{c}\text { Phase } \\
\text { de croissance } \\
\text { rapide }\end{array}$ \\
\hline $\mathrm{b}$ & 1,42 & \\
$\mathrm{~mm} \cdot \mathrm{mm}^{-1}$ par thermochrone & 0,08 & 4,18 \\
\hline Ecart-type de b & 0,93 & 0,61 \\
\hline Coefficient de détermination & 22 & 0,81 \\
\hline Nb. de données & & 13 \\
\hline \hline
\end{tabular}

D'autre part, après lissage des données élémentaires (moyenne pas à pas des données couvrant $1 / 3$ de thermochrone), on obtient l'estimée du taux de croissance «instantané » en calculant empiriquement la dérivée point par point de la courbe (fig. 12b).

\section{RÉFÉRENCES BIBLIOGRAPHIQUES}

CNRS, 1979. La physiologie de la floraison. Ed. CNRS, Paris. Halperin W., 1978. Organogenesis at the shoot apex. Annu. Rev. Physiol., 29, 239-262.

Jonard P., Koller J., Vincent A., 1952. Evolution de la tige et de l'épi chez la variété de blé Vilmorin 27 au cours de la période de reproduction. Ann. Amél. Plant., 1 (3), 256-276.

Malvoisin P.. 1984. Organogenèse et croissance du maître-brin du blé tendre (Triticum aestivum) du semis à la floraison. I. Relations observées entre la croissance foliaire et la différenciation des ébauches foliaires ou florales. Agronomie, 4 (6), 557-564

Nozeran R., 1978. Réflexions sur les enchaînements de fonctionnement au cours du cycle des végétaux supérieurs. Bull. Soc. Bot. Fr., 125, 263-280.

Nozeran R., Bancilhon L., Neville P., 1971. Intervention of internal correlations in the morphogenesis of higher plants. $A d v$. in Morphog., 9, 1-65.

Patrick J. W., $1972 a$. Vascular system of the wheat plant. I. Mature state. Aust. J. Bot., 20, 49-63.

Patrick J. W., $1972 b$. Vascular system of the wheat plant. II. Development. Aust. J. Bot., 20, 65-78

Vincent A., 1982. Morphogenèse du blé (maître-brin). Doc. ronéo groupe blé, janvier 1982.

Williams R. F., 1975. The shoot apex and leaf growth. Camb. Univ, Press, $256 \mathrm{p}$.

Williams R. F., Rijven A. H., 1965. The physiology of growth in the wheat plant. II. The dynamics of leaf growth. Aust. J. Biol. Sci., $18,722-743$ 